\title{
Antimicrobial resistance patterns in extended-spectrum $\beta$-lactamase producing Escherichia coli and Klebsiella pneumoniae isolates in a private tertiary hospital, Kenya
}

Daniel Maina ${ }^{1 *}$, Paul Makau', Andrew Nyerere ${ }^{2}$ and Gunturu Revathi ${ }^{1}$

*Correspondence: daniel.maina@aku.edu

'Department of Pathology, Aga Khan University Hospital, Nairobi, Kenya.

${ }^{2}$ Department of Medical Microbiology, Jomo Kenyatta University of Agriculture and Technology, Nairobi, Kenya.

\begin{abstract}
Background: Extended-Spectrum $\beta$-Lactamase (ESBL) producing Enterobacteriaceae have become widespread in hospitals and are increasing in community settings where they cause a variety of infections. In addition to hydrolyzing most beta-lactam agents, bacteria harboring these enzymes display resistance to other unrelated antimicrobial agents and thus often pose a therapeutic dilemma. Though these resistance patterns have been studied elsewhere within Africa, such data from hospitals in Kenya are scanty. We therefore undertook to determine these patterns at the Aga Khan University hospital by studying multidrug resistant Escherichia coli and Klebsiella pneumoniae isolates from patients' samples.

Findings: A total of 159 isolates (109 E. coli and 50 K. pneumoniae) were confirmed as ESBL producers and tested against eighteen antimicrobials. The proportion of resistant isolates was high $(>80 \%)$ for the antimicrobials tested with the exception of the carbapenems $(<1 \%)$, nitrofurantoin $(23 \%)$ and gentamicin $(63 \%)$. Klebsiella pneumoniae had a higher proportion of isolates resistant to ceftazidime, gentamicin and nitrofurantoin $(\mathrm{P}<0.05)$ than $E$. coli which had a higher proportion of isolates resistant to ciprofloxacin, levofloxacin and tetracycline $(\mathrm{P}<0.05)$.

Conclusions: In our study, antimicrobial coresistance is common in ESBL producers; however resistance to carbapenems is low and these drugs would be the appropriate empiric therapy for serious or life threatening systemic infections. Nitrofurantoin retains good activity among the multidrug resistant isolates and can be the drug of choice for non-complicated urinary tract infections due to ESBL producing E. coli.
\end{abstract}

Keywords: Extended-spectrum beta lactamases, Escherichia coli, Klebsiella pneumoniae, antimicrobial susceptibility, empiric therapy

\section{Background}

The precise definition of the term Extended-Spectrum $\beta$-Lactamase (ESBL) remains unclear but is generally used to refer to any $\beta$-lactamase, 'generally acquired rather than inherent to a species, that is either able to confer resistance to oxyimino-cephalosporins (but not carbapenems), or that has an increased ability to do so, as compared with classic members of its genetic family' [1].

ESBL producing strains of Enterobacteriaceae have emerged as a major problem in hospitalized as well as community based patients [2]. These organisms are responsible for a variety of infections like urinary tract infection (UTI), septicemia, hospital acquired pneumonia, intra-abdominal abscess, brain abscess and device related infections.

Organisms producing ESBLs are clinically relevant and remain an important cause of failure of therapy with cephalosporins [3]. ESBLs are primarily produced by the Enterobacteriaceae family, in particular Klebsiella pneumoniae and Escherichia coli [4].

Bacteria harboring ESBLs may also acquire and most often exhibit additional resistances to other antimicrobial classes such as the quinolones, tetracyclines, cotrimoxazole, trimethoprim, and aminoglycosides, which further limits therapeutic options and thus pose a therapeutic dilemma [5-8].

The Aga Khan University hospital (AKUH) is a private tertiary institution located in Nairobi, Kenya, with twenty satellite clinics spread across the country that send specimens for culture to the main laboratory. Among the Enterobacteriaceae, E. coli and $K$. pneumoniae are the two most isolated organisms from patients' samples in the hospital's microbiology section and ESBL producing isolates account for approximately $8 \%$ of the two species. Cephalosporins are among the most prescribed antibiotics in the hospital. In the year 2009 about 250,000 doses of ceftriaxone were consumed in the outpatient department alone. The antibiotic susceptibility profiles of these ESBL producing isolates have not been previously described in this hospital and are the focus of this study.

\section{Methods}

Three hundred thirty six multidrug resistant isolates cultured from clinical specimens and which were reported as $E$. coli and $K$. pneumoniae by use of routine bench biochemical tests and stored at $-80^{\circ} \mathrm{C}$ in a broth medium with glycerol during the period 2007 to 2009 were included in the study. Only one isolate per patient was included. The isolates were revived on 
Maina et al. Microbiology Discovery 2013,

http://www.hoajonline.com/journals/pdf/2052-6180-1-5.pdf

doi: $10.7243 / 2052-6180-1-5$

Table1. Distribution of clinical specimens and isolates cultured.

\begin{tabular}{lccccc}
\hline & \multicolumn{2}{c}{ Inpatient } & \multicolumn{3}{c}{ Outpatient } \\
\hline Specimen type & E. coli & K. pneumoniae & E. coli & K. pneumoniae & Total \\
\hline ABDOMINAL DRAIN & 1 & 0 & 0 & 0 & 1 \\
ASITIC FLUID & 1 & 0 & 0 & 0 & 1 \\
AXILLA SWAB & 2 & 0 & 0 & 0 & 2 \\
BRONCHIAL LAVAGE & 0 & 1 & 0 & 0 & 1 \\
FLUID & & & & & \\
BLOOD & 1 & 1 & 0 & 0 & 2 \\
CATHETER TIP & 0 & 1 & 0 & 0 & 1 \\
CSF & 1 & 0 & 0 & 0 & 1 \\
ENDOCERVICAL SWAB & 0 & 0 & 1 & 0 & 1 \\
GROIN SWAB & 1 & 0 & 0 & 0 & 1 \\
JOINT ASPIRATE & 0 & 1 & 0 & 0 & 1 \\
NASAL SWAB & 0 & 1 & 0 & 0 & 1 \\
PUS SWAB & 5 & 8 & 6 & 2 & 21 \\
SPUTUM & 2 & 3 & 0 & 1 & 6 \\
TRACHEAL ASPIRATE & 1 & 3 & 0 & 0 & 4 \\
URINE & 23 & 15 & 64 & 13 & 115 \\
Total & $\mathbf{3 8}$ & $\mathbf{3 4}$ & $\mathbf{7 1}$ & $\mathbf{1 6}$ & $\mathbf{1 5 9}$ \\
\hline & & & & &
\end{tabular}

Table2. Antibiotic susceptibilities of the isolates $n=159$.

\begin{tabular}{lcc}
\hline Antibiotic & $\begin{array}{c}\text { Resistant } \\
\text { No (\%) }\end{array}$ & $\begin{array}{c}\text { Sensitive } \\
\text { No (\%) }\end{array}$ \\
\hline Amoxicillin/Clavulanate & $159(100 \%)$ & 0 \\
Ampicillin & $159(100 \%)$ & 0 \\
Ceftazidime & $144(90.6 \%)$ & $15(9.4 \%)$ \\
Cefuroxime & $158(99.4 \%)$ & $1(0.6 \%)$ \\
Ciprofloxacin & $132(83 \%)$ & $27(17 \%)$ \\
Cefpodoxime & $158(99.4)$ & $1(0.6 \%)$ \\
Cefotaxime & $158(99.4 \%)$ & $1(0.6 \%)$ \\
Gentamicin & $101(63.6 \%)$ & $58(36.4 \%)$ \\
Imipenem & $1(0.6 \%)$ & $158(99.4 \%)$ \\
Levofloxacin & $128(80.5 \%)$ & $31(19.5 \%)$ \\
Meropenem & $1(0.6 \%)$ & $158(99.4 \%)$ \\
Nitrofurantoin & $37(23.3 \%)$ & $122(76.7 \%)$ \\
Piperacillin & $159(100 \%)$ & 0 \\
Roxithromycin & $159(100 \%)$ & 0 \\
Sulfamethoxazole/Trimethoprime & $144(90.6 \%)$ & $15(9.4 \%)$ \\
Teteracycline & $131(82.4 \%)$ & $28(17.6 \%)$ \\
Tobramycin & $131(82.4 \%)$ & $28(17.6 \%)$ \\
Tazobactam/Piperacillin & $154(96.9 \%)$ & $5(3.1 \%)$ \\
\hline & & \\
\hline
\end{tabular}

MacConkey media plates. Confirmation of ESBL production was done using the combined disc method [ceftazidime

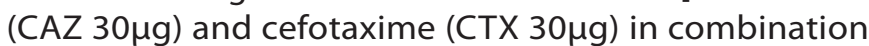

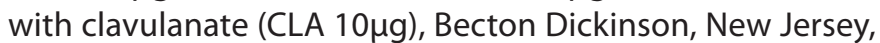
USA] as per the Clinical Laboratory Standards Institute (CLSI 2009) guidelines on Mueller Hinton with $5 \%$ sheep blood agar plates, and antibiotic susceptibility performed using VITEK 2 (version 4.01, bioMerieux, Marcy-l'Etoile, France) and interpreted using the same CLSI 2009 guidelines. Klebsiella pneumoniae K6 ATCC 700603 (ESBL producer) and Escherichia coli ATCC 25922 (non-ESBL producer) served as the positive and negative controls respectively.

Eighteen antimicrobial agents were included; amoxicillin/ clavulanate, ampicillin, ceftazidime, cefuroxime, ciprofloxacin, cefpodoxime, cefotaxime, gentamicin, imipenem, levofloxacin, meropenem, nitrofurantoin, piperacillin, roxithromycin, sulfamethoxazole/trimethoprime, teteracycline, tobramycin, and tazobactam/piperacillin.

Data were analyzed using the statistical package for social sciences (SPSS 15.0, IBM SPSS, New York, USA).

Contingency table analysis was done by a chi-square test or two-tailed Fisher's exact test where applicable. A p-value of less than 0.05 was considered as statistically significant. Ethical approval was granted by the Aga Khan University Hospital Nairobi Ethics Committee.

\section{Findings}

\section{Isolate characteristics}

Out of the 336 isolates initially included, 177 isolates could not either be revived, did not have patient details, were neither $E$. coli nor K. pneumoniae on retesting with VITEK 2, or were not ESBL producers and therefore excluded leaving 159 isolates.

A hundred and nine of the isolates (68.6\%) were E. coli and 50 (31.4\%) were K. pneumoniae. Outpatient specimens were 87 (45.3\%) and inpatient 72 (45.3\%). Escherichia coli were isolated more frequently in outpatients than $K$. pneumoniae $(p<0.05)$.

Urine was the most common specimen processed with 115 (72\%) of the samples. More than half of the urine samples were from outpatients. Other specimens were fewer and mostly from in-patients (Table 1). The ages of patients from whom the specimens were recovered ranged from 2 days to 90 years with a median of 42 years.

\section{Antibiotic susceptibility patterns}

A large proportion of the isolates ( $>80 \%$ ) were resistant to all classes of antibiotics tested apart from the carbapenems to which resistance was found in a single isolate. The proportion of isolates resistant to nitrofurantoin was substantially lower at $23 \%$ and $63 \%$ for gentamicin (Table 2).

There were significant species specific differences in the resistance patterns to some antibiotics. Klebsiella pneumoniae had a higher proportion of isolates resistant to ceftazidime, gentamicin and nitrofurantoin than E. coli isolates. In turn E. coli had a higher proportion of isolates resistant to ciprofloxacin, levofloxacin and tetracycline (Table 3).

\section{Discussion}

Our study analyzed 159 isolates from in-patients and outpatients, thus encompassing both hospital and community acquired infections due to ESBL producing $E$. coli and $K$. pneumoniae.

The two bacteria species studied had high proportions of resistant isolates to most antimicrobial agents with the exception of carbapenems and nitrofurantoin.

Similar findings have been reported in other studies in Iran and Tanzania $[\mathbf{9 , 1 0}]$. In these two studies imipenem was shown to be the most active antibiotic against the ESBL producing isolates and nitrofurantoin showed moderate activity. 
Maina et al. Microbiology Discovery 2013,

Table3. Significant species specific differences in antimicrobial resistance.

\begin{tabular}{lcccc}
\hline Antibiotic & Sensitivity & E. coli $(\mathbf{n}=\mathbf{1 0 9})$ & K.pneumoniae $(\mathbf{n}=\mathbf{5 0})$ & P-value \\
\hline Ceftazidime & $\mathrm{R}$ & 95 & 49 & 0.038 \\
& $\mathrm{~S}$ & 14 & 1 & \\
Ciprofloxacin & $\mathrm{R}$ & 101 & 31 & $<0.001$ \\
& $\mathrm{~S}$ & 8 & 19 & \\
Levofloxacin & $\mathrm{R}$ & 99 & 29 & $<0.001$ \\
\multirow{3}{*}{ Nitrofurantoin } & $\mathrm{S}$ & 10 & 21 & \\
& $\mathrm{R}$ & 12 & 25 & $<0.001$ \\
Tetracycline & $\mathrm{S}$ & 97 & 25 & 0.005 \\
& $\mathrm{R}$ & 96 & 35 &
\end{tabular}

$\mathrm{R}=$ resistant, $\mathrm{S}=$ sensitive

The finding that nitrofurantoin remains active in the majority of isolates is clinically important to the outpatient setting, where most of the isolates from urine tended to be $E$. coli or K. pneumoniae.

Nitrofurantoin can therefore be used effectively for most non-life threatening urinary tract infections with little regard to the antibiotic resistance mechanisms at play among ESBL producing isolates. From our experience many clinicians prefer to prescribe other drugs yet nitrofurantoin remains effective in most cases where there are multiple resistances as is also evident in other studies $[11,12]$.

Given the popularity of fluoroquinolones in treating a variety of infections including respiratory, gastrointestinal and urinary tract infections, the two drugs tested (ciprofloxacin and levofloxacin) fared poorly with $>80 \%$ of the isolates showing resistance to both. This is not surprising among ESBL producers and this phenomenon is well described in the literature. The CTX-M genotype is the one that has most been associated with this resistance $[6,13]$.

The CTX-M genotype has been documented locally. A previous study done in this University hospital using part of the 159 isolates found the CTX-M genotype the most predominant being present in 46 (88.5\%) of the $52 \mathrm{ESBL}$ isolates tested [14]. It has also been suggested that the use of fluoroquinolones selects for and results in the spread of this genotype [15].

The resistance to carbapenems was low in our study and the single isolate that displayed resistance to the two drugs in this class needs further investigation to determine the probable additional mechanism of resistance. While this class of drugs remains useful for treating serious infections due to multidrug resistant enteric bacteria in our set up, the single isolate resistant to carbapenems is clinically relevant and suggests that this pattern of resistance should be monitored closely by microbiology laboratories.

The clinical utility of cephalosporins even with the evidence of in vitro activity in isolates that are ESBL producers remain controversial. The CLSI guidelines state they should be reported as resistant, a position that is held by some researchers while others suggest that there may be a limited clinical role for cephalosporins with activity against ESBL producers [16]. Our data suggests that cephalosporins would be of limited use in the treatment of ESBL related infections in our institution as the proportion of resistant isolates was high. These observations also apply to the $\beta$-lactam/ $\beta$-lactam inhibitor combinations for which we observed a high proportion of isolates with MICs in the resistant range. A possible explanation for the latter observation is the presence of additional resistance mechanisms which as noted above may be acquired together with ESBLs. However, these additional mechanisms were not specifically looked for in this study.

The screening for ESBL production is not routinely done in many clinical laboratories in this country and the impact of treatment failure associated with these infections when treated with cephalosporins is not well documented.

\section{Conclusions}

ESBL producing E. coli and K. pneumoniae display multiple resistances to various classes of antimicrobials and are therefore important to identify and treat accordingly. In our study the carbapenems and nitrofurantoin retain good activity and would thus be the drugs of choice for empiric therapy in life threatening infections and non-complicated urinary tract infections respectively. There is need to carry out more studies to determine the epidemiology of ESBL related infections and the associated clinical burden locally.

\section{Competing interests}

The authors declare that they have no competing interests.

\section{Authors' contributions}

DM worked on the selection of isolates, data analysis and drafted the manuscript. PM was involved in study design and data analysis. AN provided technical input on the bench and reviewed the susceptibilities data. GR conceived of the study, gained ethical approval and coordinated the study. All the authors read and approved the final manuscript.

\section{Acknowledgement}

We are grateful to the Aga Khan University for granting permission and the funds to carry out this study.

\section{Publication history}

Received: 14-Jan-2013 Revised: 04-Mar-2013

Accepted: 25-Mar-2013 Published: 03-Apr-2013

\section{References}

1. Livermore DM: Defining an extended-spectrum beta-lactamase. Clin Microbiol Infect 2008, 14 Suppl 1:3-10. | Article | PubMed

2. Rodriguez-Bano J, Navarro MD, Romero L, Martinez-Martinez L, Muniain MA, Perea EJ, Perez-Cano R and Pascual A: Epidemiology and clinical features of infections caused by extended-spectrum beta-lactamaseproducing Escherichia coli in nonhospitalized patients. J Clin Microbiol 2004, 42:1089-94. | Article | PubMed Abstract | PubMed Full Text

3. Bradford PA: Extended-spectrum beta-lactamases in the 21st century: characterization, epidemiology, and detection of this important resistance threat. Clin Microbiol Rev 2001, 14:933-51, table of contents. | Article | PubMed Abstract | PubMed Full Text

4. Paterson DL and Bonomo RA: Extended-spectrum beta-lactamases: a clinical update. Clin Microbiol Rev 2005, 18:657-86. | Article I PubMed Abstract | PubMed Full Text 
Maina et al. Microbiology Discovery 2013,

5. Chopra I, Schofield C, Everett M, O'Neill A, Miller K, Wilcox M, Frere JM, Dawson M, Czaplewski L, Urleb U and Courvalin P: Treatment of health-care-associated infections caused by Gram-negative bacteria: a consensus statement. Lancet Infect Dis 2008, 8:133-9. | Article | PubMed

6. Morosini MI, Garcia-Castillo M, Coque TM, Valverde A, Novais A, Loza E, Baquero $\mathrm{F}$ and Canton R: Antibiotic coresistance in extended-spectrumbeta-lactamase-producing Enterobacteriaceae and in vitro activity of tigecycline. Antimicrob Agents Chemother 2006, 50:2695-9. | Article | PubMed Abstract | PubMed Full Text

7. Pitout JD and Laupland KB: Extended-spectrum beta-lactamaseproducing Enterobacteriaceae: an emerging public-health concern. Lancet Infect Dis 2008, 8:159-66. | Article | PubMed

8. Talbot GH: What is in the pipeline for Gram-negative pathogens? Expert Rev Anti Infect Ther 2008, 6:39-49. | Article | PubMed

9. Mehrgan $H$, Rahbar M and Arab-Halvaii Z: High prevalence of extendedspectrum beta-lactamase-producing Klebsiella pneumoniae in a tertiary care hospital in Tehran, Iran. J Infect Dev Ctries 2010, 4:132-8. | Article I PubMed

10. Moyo SJ, Aboud S, Kasubi M, Lyamuya EF and Maselle SY: Antimicrobial resistance among producers and non-producers of extended spectrum beta-lactamases in urinary isolates at a tertiary Hospital in Tanzania. BMC Res Notes 2010, 3:348. | Article | PubMed Abstract | PubMed Full $\underline{\text { Text }}$

11. Garau J: Other antimicrobials of interest in the era of extendedspectrum beta-lactamases: fosfomycin, nitrofurantoin and tigecycline. Clin Microbiol Infect 2008, 14 Suppl 1:198-202. | Article | PubMed

12. Schito GC, Naber KG, Botto H, Palou J, Mazzei T, Gualco L and Marchese A: The ARESC study: an international survey on the antimicrobial resistance of pathogens involved in uncomplicated urinary tract infections. Int J Antimicrob Agents 2009, 34:407-13. | Article | PubMed

13. Paterson DL, Mulazimoglu L, Casellas JM, Ko WC, Goossens H, Von Gottberg A, Mohapatra S, Trenholme GM, Klugman KP, McCormack JG and Yu VL: Epidemiology of ciprofloxacin resistance and its relationship to extended-spectrum beta-lactamase production in Klebsiella pneumoniae isolates causing bacteremia. Clin Infect Dis 2000, 30:473-8. | Article | PubMed

14. Maina D, Revathi G, Kariuki S and Ozwara H: Genotypes and cephalosporin susceptibility in extended-spectrum beta-lactamase producing enterobacteriaceae in the community. J Infect Dev Ctries 2012, 6:470-7. | Article | PubMed

15. Ben-Ami R, Schwaber MJ, Navon-Venezia S, Schwartz D, Giladi M, Chmelnitsky I, Leavitt A and Carmeli Y: Influx of extended-spectrum beta-lactamase-producing enterobacteriaceae into the hospital. Clin Infect Dis 2006, 42:925-34. | Article | PubMed

16. Ramphal $R$ and Ambrose PG: Extended-spectrum beta-lactamases and clinical outcomes: current data. Clin Infect Dis 2006, 42 Suppl 4:S164-72. | Article | PubMed

\section{Citation:}

Maina D, Makau P, Nyerere A and Revathi G:

Antimicrobial resistance patterns in extended-spectrum $\beta$-lactamase producing Escherichia coli and Klebsiella pneumoniae isolates in a private tertiary hospital, Kenya. Microbiology Discovery 2013, 1:5.

http://dx.doi.org/10.7243/2052-6180-1-5 